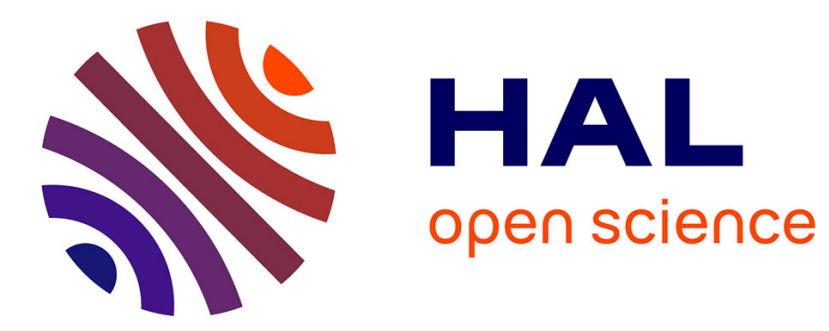

\title{
Plasmonic nanosponges filled with silicon for enhanced white light emission
}

\author{
A. Larin, A. Nomine, E. Ageev, J. Ghanbaja, L. Kolotova, S. Starikov, S. \\ Bruyère, T. Belmonte, S. Makarov, D. Zuev
}

\section{- To cite this version:}

A. Larin, A. Nomine, E. Ageev, J. Ghanbaja, L. Kolotova, et al.. Plasmonic nanosponges filled with silicon for enhanced white light emission. Nanoscale, 2020, 12 (2), pp.1013-1021. 10.1039/C9NR08952G . hal-02548983

\section{HAL Id: hal-02548983 \\ https://hal.science/hal-02548983}

Submitted on 8 Nov 2020

HAL is a multi-disciplinary open access archive for the deposit and dissemination of scientific research documents, whether they are published or not. The documents may come from teaching and research institutions in France or abroad, or from public or private research centers.
L'archive ouverte pluridisciplinaire HAL, est destinée au dépôt et à la diffusion de documents scientifiques de niveau recherche, publiés ou non, émanant des établissements d'enseignement et de recherche français ou étrangers, des laboratoires publics ou privés. 


\title{
Plasmonic Nanosponges Filled by Silicon for Enhanced White Light Emission $\dagger$
}

\author{
A. O. Larin, ${ }^{\text {a }}$ A. Nominé, ${ }^{\mathrm{a} ; \mathrm{b}}$ E. I. Ageev, ${ }^{\mathrm{a}}$ J. Ghanbaja, ${ }^{\mathrm{b}}$ L. Kolotova, ${ }^{\mathrm{c} ; \mathrm{d}}$ S. Starikov, ${ }^{\mathrm{c} ; \mathrm{e}}$ S. \\ Bruyère, ${ }^{b}$ T. Belmonte, ${ }^{b}$ S. V. Makarov, ${ }^{a}$ and D. A. Zuev ${ }^{a}$ \\ ${ }^{a}$ Department of Nanophotonics and Metamatarials, ITMO University, 49 Kronverkskii pr., \\ Saint Petersburg 197101, Russia. \\ ${ }^{\mathrm{b}}$ Institut Jean Lamour - CNRS - Université de Lorraine, Nancy, France \\ ${ }^{\mathrm{c}}$ Joint Institute for High Temperatures, Russian Academy of Sciences, Moscow 125412 , \\ Russia \\ ${ }^{\mathrm{d}}$ Moscow Institute of Physics and Technology (State University), Dolgoprudny 141700, \\ Russia \\ ${ }^{\mathrm{e}}$ Ruhr-Universität Bochum, Bochum 44801, Germany
}




\begin{abstract}
Plasmonic nanosponges is a powerful platform for various nanophotonic applications owing to extremely high local field enhancement in the metallic nanoporous. Filling of the nanoporius by semiconductors open up opportunities for enhancement of nonlinear effects in these materials. But this task remains challenging due to lack of knowledge on the integration process of metal and semiconductor components as well as internal structure of such nanoobjects. Here, we investigate metal-dielectric ( $\mathrm{Au}-\mathrm{Si}$ ) nanoparticles fabricated by the laser printing technique via combination of theoretical and experimental methods. We reveal that these hybrid nanoparticles represent the $\mathrm{Au}$ sponge nanostructure filled with $\mathrm{Si}$ nanocrystallites. We also demonstrate that the Au net provides strong near-field enhancement in Si grains increasing white light photoluminescence in the sponge nanostructures compared to uniform Si nanoparticlese. These results pave the way for engineering of the internal structure of the sponged-like hybrid nanoparticles and control of optical properties of white light luminescent nanoparticles on demand.
\end{abstract}




\section{Introduction}

Metal nanoscale frameworks, also called "nanosponges", have become a powerful tool for various applications in modern plasmonics owing to their ability to support localized plasmons and as a consequence enhance local optical field in a whole their volume. This unique property allows for avoiding the limitation of thin skin-layer in metals, which prevents the application of sub- mm plasmonic particles, and obtaining strong spatial and spectral localization resulting in exceptionally high Purcell factors on the order of $10^{62}$. Moreover, very developed internal surface of the nanosponges provides an outstanding platform for SERS $^{3,4}$, fluorescence enhancement ${ }^{5}$, as well as electrons nanoscale emission ${ }^{6}$.

As plasmonic nanosponges are fabricated from various metals and metal alloys, their proved integration with semiconductors and understanding underlying processes is a serious problem. Such unification represents especial interests, because high refractive index semiconductors and nanostructures made of these materials have recently emerged as a novel direction in nanophotonics (all-dielectric nanophotonics) expanding functionalities of plasmonics ${ }^{7-9}$. In particular filling of pores of plasmonic nanosponges by high-refractive index semiconductors can be used to enhance inherent non-linear effects and potentially provide interesting optical properties.

On the way of integration of plasmonics with the highrefractive dielectics, hybrid $\mathrm{Si} / \mathrm{Au}(\mathrm{Ag})$ nanostructures with separated metallic and semiconductor parts were recently applied for surface coloring $^{10}$, near-field and far-field tuning ${ }^{11}$, optical harmonics generation ${ }^{12}$, and fluorescence enhancement ${ }^{13}$. However, standard top-down approaches, combining these elements within a single nano-objects, are complicated and often restricted by the material properties. For instance, the synthesis of Si/Au nanoparticles (NPs) is confronted with two major physical limitations, namely the low solubility of the elements and the dewetting ${ }^{14}$ of metals on the surface of semiconductors. From this point of view non-equilibrium processes using lasers attract an increasing attention as they can make it possible to overcome some limitations imposed by thermodynamics and therefore widens the possibilities in nanofabrication for the creation of hybrid metal dielectric nanosponges.

In this paper, we employ ultrafast laser ablation to fabricate metal-dielectric NP from twolayer Si/Au films. The studies of internal composition of such nanostructures demonstrate that they represent plasmonic 'nanosponges' filled by nanocrystalline silicon. Our atomistic modeling explains creation of this complicated nanostructure representing very developed net of gold covering isolated silicon nanograins by the high cooling rates (up to $10^{6} \mathrm{Ks}^{-1}$ ) which can be achieved through laser-assisted fabrication process. We also show that the formed nanosponge supporting strong near-field localization demonstrate enhanced whitelight photoluminescence (PL) driven by near-IR laser pulses, as shown schematically in Fig. 1. Such efficient nanoscale white-light sources are prospective tools for advanced nanospectroscopy, i.e. for broadband probing of the near fields of nanophotonic devices or mapping their optical properties ${ }^{15,16}$.

\section{Results}

\subsection{Structure configurations}

Sponge NPs were fabricated by femtosecond laser ablation in the laser transfer configuration, when the ablated NPs fall on a glass substrate ${ }^{17,18}$ (for details, see Methods). Fig. 2 shows that the obtained NPs size ranges from tens to hundreds of nanometers (Fig. 2a). These NPs present the particularity to have a granular structure with clearly defined Si-rich and Au-rich grains. This is intuitively visible from the contrast of scanning transmission electron 
microscopy (STEM) images and confirmed by energydispersive X-ray spectroscopy (EDS) mapping of NPs with 135 and $195 \mathrm{~nm}$ diameters as shown in Fig. 2a. However, the configuration of the NPs, with overlapping of grains of different composition, makes it difficult to accurately determine the composition of each phase. High-Resolution TEM (HRTEM) images in Fig. 2b show that both phases are crystallized. The analysis of interplanar distances (dhkl) shows that NPs are composed of a cubic diamond structure with a lattice parameter of $0.543 \mathrm{~nm}$ and a face centered cubic structure with a lattice parameter of $0.409 \mathrm{~nm}$ which is consistant with silicon and gold respectively. Details of the calculation are given in the ESI, Section 1.

Microgaphs presented in Fig. 2(a,b) give only a partial insight of the microstructure of NPs. To overcome this limitation, a cross-sectional sample of NPs was prepared by means of focused ion beam (FIB). EDS mapping of the cross-sectional sample (Fig. 2(c)) reveals a network of gold within a silicon matrix. Generally, the applied advanced techniques of structural characterization (for details, see Methods) allowed for observe and study such sponge-like NPs made of metal and semiconductor for the first time to our knowledge. Therefore, detailed theoretical analysis is required to describe the main dependencies of the internal structure on composition and size of NPs.

\subsection{Modeling of NPs composition}

In this work, we performed the atomistic simulations of Si/Au NP cooling with various values of the cooling rate $K$ and different gold content (Au content was variated from 0 to 30 atomic percent). The interactions between atoms were described by the angular-dependent interatomic potential developed on the basis of ab initio calculations ${ }^{19}$.

According to previous works ${ }^{19,20}$, the final structure of the formed silicon-based NPs strongly depends on the cooling rate $\mathrm{K}=\mathrm{dT} / \mathrm{dt}$. Crystallization of NP takes place in the case, when its cooling rate is smaller than the threshold one $\mathrm{K}_{\mathrm{th}}$. The threshold cooling rates for the crystallization processes at various NP compositions were calculated in the previous work ${ }^{19}$.

The atomistic simulations of internal structure of the formed Si/Au NPs are shown in the Fig. 3. First of all, the smaller the cooling rate, the larger average size of the formed crystallines in NP. moreover, $\mathrm{Au}$ is almost insoluble in $\mathrm{Si}$ and increasing of $\mathrm{Au}$ content leads to decrease in liquidus temperature $T_{\text {liq }}{ }^{21}$. Due to this fact, $\mathrm{Au}$ atoms tend to leave crystallization area via atomic diffusion. Therefore, most of $\mathrm{Au}$ atoms are concentrated on the crystalline grain boundaries and on NPs surface, and the formed crystallines contains about $\sim 5$ atomic percent of Au inside. This effect is more pronounced at high gold concentrations 20-30 atomic percent and lower cooling rates (Fig. 3a,b). In turn, at low Au concentrations 5-10 atomic percent and high cooling rates, $\mathrm{Au}$ concentration on the crystalline grain boundaries is approximately equal to the Au concentration inside the crystallines (Fig. 3c). According to our simulations, the growth of Au concentration in NP results in increase of the fraction of $\mathrm{Au}$ atoms on the grain boundaries as compared with the fraction of Au inside the grains (Fig. 4). Also, increasing of Au concentration results in a decrease of $\mathrm{K}_{\mathrm{th}}$ for the fixed NP size.

In order to compare the atomistic simulations for $\mathrm{Si} / \mathrm{Au}$ NPs with the available experimental data, estimation of the cooling rate $\mathrm{K}$ in realistic conditions is necessary. Such detailed description of cooling regimes of pure silicon NPs was carried out in our previous work ${ }^{20}$. According to this model, $\mathrm{T}$ of moving NP on distance $\mathrm{z}$ may be estimated as: 


$$
\begin{gathered}
T \approx T_{0}-K \cdot \tau \ln \frac{v_{0} \tau}{v_{0} \tau-z} \\
K \approx K_{0} \cdot(50 / R) \cdot \frac{\rho_{\mathrm{si}}}{\rho_{\mathrm{np}}}
\end{gathered}
$$

where $\mathrm{K}_{0}=0: 009 \mathrm{~K} / \mathrm{ps}, \rho_{\mathrm{si}}$ is the atomic silicon density and $\rho_{\mathrm{np}}$ is the atomic $\mathrm{Si} / \mathrm{Au} \mathrm{NP}$ density, $\mathrm{R}$ is the NP radius in nanometer unit, $\tau=2 \rho_{n p} \mathrm{R}^{2} / 9 \eta$ is relaxation time. The crystallization of NP takes place at temperature $\mathrm{T} \sim 0.75 \mathrm{~T}_{\text {liq }}{ }^{19}$, if the cooling rate $\mathrm{K}$ is less than $\mathrm{K}_{\mathrm{th}}$. In this case, the radius of the NP that crystallizes in the air can be found as:

$$
R_{u p}^{2} \approx R_{0}^{2} \cdot \frac{\rho_{\mathrm{si}}}{\rho_{\mathrm{np}}} \cdot\left(1-\exp \left(\frac{0.75 T_{l i q}-T_{0}}{t_{R} R_{u p}}\right)\right)^{-1}
$$

where $\mathrm{R}_{0}^{2}=9 \eta \mathrm{z} /\left(2 \rho_{\mathrm{si}} \mathrm{v}_{0}\right), \mathrm{z}$ is the distance between substrates and $t_{\mathrm{R}}=100 \mathrm{~K}_{0} \rho_{\mathrm{si}}=9 \eta$. $\mathrm{T}_{\text {liq }}$ and $\rho_{\text {np }}$ depend on the Au concentration. The cooling rate of NP on a substrate is close to the threshold cooling rate $\mathrm{K}_{\mathrm{th}}$, and if NP reaches the substrate in the liquid state it becomes amorphous $^{20}$. The formula (2) allows us to roughly estimate the upper limit for NP size for which crystallization is possible (line 1 on Figure 5). On the other hand, the threshold cooling rate $\mathrm{K}_{\mathrm{th}}$ decreases with the increasing of $\mathrm{Au}$ concentration. This leads to the fact that for small values of NP radius actual cooling rate $K$ in the air becomes larger than $K_{t h}$. So the obtained $\mathrm{NP}$ are amorphous anyway as it is observed in the experiment. Thus, the lower limit of crystallization possibility can be is found from condition $\mathrm{K} \sim \mathrm{K}_{\mathrm{th}}$. As a result:

$$
R_{\text {down }} \approx 50 \cdot \frac{K_{0}}{K_{t h}} \cdot \frac{\rho_{\mathrm{si}}}{\rho_{\mathrm{np}}}
$$

Fig. 5 summarizes the estimation of NP radius R and Au concentration that correspond to NP crystallization. The position of lines 1 and 2 depends on initial parameters in Eq.(2) and Eq.(3). Fig. 5 corresponds to the following parameters: $T_{0}=1900 \mathrm{~K} ; \mathrm{v}_{0}=100 \mathrm{~m} / \mathrm{s}$, the distance $\mathrm{z}=40 \mathrm{~mm}$. Also, the average grain size in the NP depends on the cooling rate (Fig. $3 d$ ), which in turn depends on the NP radius and Au concentration as following $K \sim\left(\rho_{n p} R\right)^{-1}$. Thus, in theory, the average grain size decreases with Au concentration increasing (Fig. 5). Moreover, the average grain size can be estimated from the experimental data (see Fig.S3 in Supporting information), corresponding to the points on Fig. 5.

\subsection{White light emission}

To understand influence of internal composition of hybrid nanosponges on the optical properties, they were studied numerically and experimentally. Nonlinear PL measurements were conducted for the hybrid sponge nanoparticles by irradiation of fs-laser pulses $(\lambda=1050$ $\mathrm{nm}$ or $1.18 \mathrm{eV}, \tau=150 \mathrm{fs}$ ) with intensity of $50 \mathrm{GW} / \mathrm{cm}^{2}$. The Fig. 6a demonstrates typical PL spectrum from hybrid nanosponge and $\mathrm{Si}$ nanoparticle excited near resonant conditions. The spectrum of a $150 \mathrm{~nm}$ hybrid nanoparticle fabricated from $60 / 15 \mathrm{~nm} \mathrm{Si} / \mathrm{Au}$ film is extremely strong and covers the entire visible and part of near-infrared spectrum with a characteristic white-color photoluminescence as well as second-harmonic (SHG) signal at $525 \mathrm{~nm}$. The less intense PL signal was observed for a 300-nm Si NP fabricated from the 50- nm a-Si:H film by the laser printing method. Thus, hybrid sponge nanoparticles provide white light luminescence signal up to 20 times higher compared to silicon ones ${ }^{16}$. To explain this effect 
we have conducted numerical modeling of optical properties of hybrid nanosponge taking into account internal composition of the nanostructure. According to the modeling, absorption cross- section for the hybrid nanosponge is several times stronger at the photoexcitation wavelength $(\lambda=1050 \mathrm{~nm})$ (see Fig. 6b). Moreover, the modeling of near-field inside the sponge nanoparticle shows strong local field enhancement in silicon parts as well (Fig. 6c). To analyze accumulated energy of the incident light in the silicon component of both sponge and silicon NPs, the time-averaged absolute value of electric field $|E|$ over the volume of silicon was calculated using a square grid with a step of $1 \mathrm{~nm}$ following by summation of all points of the electric field in the silicon volume. This numerical integral was calculated for the field in the first $(n=1)$, second $(n=2)$, fourth $(n=4)$ and sixth $(n=6)$ degrees: $\int_{\mathrm{Si}}|\mathrm{E}|^{\mathrm{n}} \mathrm{dV}$ (see Fig. $6 \mathrm{~d})$. In case of the first $(n=1)$ and second $(n=2)$ degrees, the value of the integral is larger for the Si NP. However, for the integrals with the fourth $(n=4)$ and sixth $(n=6)$ degrees, the $E$ field integral for Si/Au NPs turns out to be several orders of magnitude larger: $n=4-$ in 4.9 times, $n=6-$ in 4400 times. These full-wave optical simulations help to reveal the origin of the strong PL enhancement shown in Fig. 6a, because its nonlinear dependence on the intensity of incident near-IR laser pulse $\mathrm{I}_{\mathrm{PL}} \sim 1^{3}$, as proven in ${ }^{16}$. The absorbed energy at $\lambda=1050 \mathrm{~nm}$ is converted to the hot electrons via three-photon absorption both in silicon and gold $^{22}$. The electrons and holes generated in gold after such interband (s-d zones) and intraband (within s zone) transitions can tunnel into silicon. As a result, the hot electron-hole pairs in silicon relax nonradiatively and recombine by emitting photons. Observed broadband stationary emission indicates efficient PL of hot charge carriers ${ }^{16}$.

\section{Conclusion}

We have shown and studied novel nanophotonic design representing a golden net of nanostructures wrapping silicon nanograins. With high-resolution TEM imaging, atomistic simulations, and numerial simulations, we have revealed the origin of the observed strong nonlinear PL generation from this unique configuration. As a result, our findings will help to optimize this novel tipe of nano-objects used for a broadband near-field microspectroscopy. We have developed and realized a novel nanophotonic design representing a net of golden nanostructures wrapping silicon nanograins (sponge nanostructure). Analysis by the highresolution electron microscopy is conducted representing data about internal configuration of the nanosponges fabricated by the femtosecond laser printing method. Numerical modeling by the molecular dynamics method provides estimations of the cooling rate of the nanostructure after femtosecond laser ablation as well as possible mechanisms for creation of the final nanostructure. The broadband photoluminescence capturing both visible and near-infrared ranges is demonstrated when the nanostructures are irradiated by the femtosecond laser at the wavelength of $1050 \mathrm{~nm}$. We demonstrate that the hybrid sponge nanostructures showed a significant increase in the efficiency of photoluminescence emission compared to silicon nanoparticle, in particular, due to the localization of the electric field by the plasmonic component of the nanostructure which is supported by our numerical modelling. The obtained data provides new data about physics of this novel type of nanoobjects and open the way for creation ultracompact sources of white light for application in the near-field microscopy, lab-on-a-chip systems, sensors, etc.

\section{Methods}

\subsection{Nanoparticles fabrication}


$\mathrm{Si} / \mathrm{Au}$ NPs were fabricated using the femtosecond laser ablation in air at room temperature (294 K). Bilayer films $(15 \mathrm{~nm} / 60 \mathrm{~nm} \mathrm{Au} / \mathrm{Si})$ on a glass substrate were employed as targets for ablation. As a source of ultrashort laser pulses we used a commercial system TEMA-150 (Avesta project) operating at wavelength $1050 \mathrm{~nm}$, spectral width $7 \mathrm{~nm}$, pulse duration $150 \mathrm{fs}$, repetition rate $1 \mathrm{kHz}$ after a pulse peaker. Laser radiation with approximately $50 \mathrm{~nJ}$ per pulse was focused using Mitutoyo Plan Apo NIR Infinity Corrected Objective (10x, NA = 0.26). In the process of laser transfer of NP, the Au/Si bilayer target was placed on the distance $40 \mu \mathrm{m}$ from a receiving surface (glass or TEM grid).

\subsection{Structure characterization}

NPs were further characterized by transmission electron microscopy (TEM) using a JEOL ARM 200F Cold FEG TEM/STEM operating at $200 \mathrm{kV}$ and equipped with a spherical aberration (Cs) probe corrector with a $0.19 \mathrm{~nm}$ point resolution in TEM mode and $0.078 \mathrm{~nm}$ in Scanning mode (STEM).

\subsection{Numerical simulations of molecular dynamics}

The simulations of NPs composition were carried out using the LAMMPS $\operatorname{code}^{23}$ in the quasi2D case: one of the sizes in the calculation cell was about $3.5 \mathrm{~nm}$, and periodic boundary conditions were used. Therefore, the simulated system consists of a single nanowire with a variable diameter from $20 \mathrm{~nm}$ to $60 \mathrm{~nm}$. Random distribution of atoms ( $\mathrm{Si}$ and $\mathrm{Au}$ ) in the nanowire was used to produce the necessary Au concentration. The initial temperature of nanoparticle was equal to $2000 \mathrm{~K}$. Then the liquid nanowire was cooled down to room temperature using of the Langevin thermostat.

\subsection{Photoluminescence measurements}

Nonlinear optical microscopy was employed for the single nanoparticles nonlinear PL measurements. Laser pulses emitted by $\mathrm{Yb}^{+3}$ laser (TeMa, Avesta Project) are monitored by a compact spectrometer (ASP-75, Avesta Project) and autocorrelator (AA-20DD, Avesta Project). Energy of laser pulses is controlled by an attenuatorm and monitored before the focusing objective by a powermeter (Ophir). Optical images of emitting hybrid nanoparticles on a silica substrate are formed by using a collecting objective and CCD camera. The second optical channel allows for simultaneous spectrum recording with a spectrometer (Horiba LabRam HR). Spectral sensitivity of the CCD in the spectrometer enables spectral measurement in the range of 200-1100 nm. The efficiency of PL in forward direction is estimated by dividing the incident power at fundamental wavelength $(1050 \mathrm{~nm})$ to output PL power collected by the x 50 objective with taking into account the used optical filters KG-3 and KG-5 (Edmund Optics).

\subsection{Numerical simulations of optical properties}

Numerical calculations of the model for the sponge-type Si/Au NP and a silicon sphere were calculated using finite integration technique from CST Microwave Studio software. The system was excited by a plane wave with linear polarization and with an electric field amplitude of $1 \mathrm{~V} / \mathrm{m}$. We used the dielectric permeability data for Au and Si from the articles ${ }^{24}$ and $^{25}$, respectively. The geometry of the Si NP is a sphere with a radius of $150 \mathrm{~nm}$. For $\mathrm{Si} / \mathrm{Au} \mathrm{NP}$, the geometry was set as follows: the main particle is a silicon sphere with a radius of $150 \mathrm{~nm}$, with small fractions of the gold inside. Each Au sphere has a radius in the range of $16-36 \mathrm{~nm}$ and the position of the center of the particle does not extend beyond the boundaries 
of the Si sphere. The size and position of the Au inclusions were determined by a random number generator and a uniform probability distribution. The $\mathrm{Au}$ spheres can intersect with each other. In this configuration, the ratio of Au to Si volumes is taken as 13:20.

\section{Acknowledgements}

This work was supported by Russian Foundation for Basic Research (17-02-00538 and 1629-05317). "S.V.M. acknowledges the Ministry of Education and Science of the Russian Federation (Project 14.Y26.31.0010) for supporting the nonlinear optical experiments." DZ acknowledges the Ministry of Education and Science of Russian Federation (Project 2.2267.2017/4.6) 


\section{References}

1 D. Wang and P. Schaaf, Advances in Physics: X, 2018, 3, 1456361.

2 J. Zhong, A. Chimeh, A. Korte, F. Schwarz, J. Yi, D. Wang, J. Zhan, P. Schaaf, E. Runge and C. Lienau, Nano letters, 2018, 18, 4957-4964.

3 L. Qian, B. Das, Y. Li and Z. Yang, Journal of Materials Chemistry, 2010, 20, 6891-6895.

4 S. Syubaev, A. Nepomnyashchiy, E. Mitsai, E. Pustovalov, O. Vitrik, S. Kudryashov and A. Kuchmizhak, Applied Physics Letters, 2017, 111, 083102.

5 C. Vidal, D. Sivun, J. Ziegler, D. Wang, P. Schaaf, C. Hrelescu and T. A. Klar, Nano letters, 2018, 18, 1269-1273.

6 G. Hergert, J. Vogelsang, F. Schwarz, D. Wang, H. Kollmann, P. Groß, C. Lienau, E. Runge and P. Schaaf, Light: Science \& Applications, 2017, 6, e17075.

7 A. Krasnok, S. Makarov, M. Petrov, R. Savelev, P. Belov and Y. Kivshar, Metamaterials X, 2015, p. 950203.

8 A. I. Kuznetsov, A. E. Miroshnichenko, M. L. Brongersma, Y. S. Kivshar and B. Lukâ־A 'Zyanchuk, Science, 2016, 354, aag2472.

9 S. Jahani and Z. Jacob, Nature nanotechnology, 2016, 11, 23. 10 D. A. Zuev, S. V. Makarov, I. S. Mukhin, V. A. Milichko, S. V. Starikov, I. A. Morozov, I. I. Shishkin, A. E. Krasnok and P. A. Belov, Advanced Materials, 2016, 28, 3087-3093.

11 Y. Sun, I. Sinev, A. Zalogina, E. Ageev, H. Shamkhi, F. Komissarenko, I. Morozov, S. Lepeshov, V. Milichko, S. Makarov et al., Laser \& Photonics Reviews, 2019, 13, 1800274.

12 T. Shibanuma, G. Grinblat, P. Albella and S. A. Maier, Nano letters, 2017, 17, 2647-2651.

13 N. Livneh, M. G. Harats, S. Yochelis, Y. Paltiel and R. Rapaport, Acs Photonics, 2015, 2, 1669-1674.

14 J. Ye, D. Zuev and S. Makarov, International Materials Reviews, 2018, 1-39.

15 H. A. Bechtel, E. A. Muller, R. L. Olmon, M. C. Martin and M. B. Raschke, Proceedings of the National Academy of Sciences, 2014, 111, 7191-7196.

16 S. V. Makarov, I. S. Sinev, V. A. Milichko, F. E. Komissarenko, D. A. Zuev, E. V. Ushakova, I. Mukhin, Y. Yu, A. I. Kuznetsov, P. A. Belov et al., Nano letters, 2017, 18, 535539.

17 A. Kuznetsov, J. Koch and B. Chichkov, Optics express, 2009, 17, 18820-18825.

18 P. Dmitriev, S. V. Makarov, V. Milichko, I. Mukhin, A. Gudovskikh, A. Sitnikova, A. Samusev, A. Krasnok and P. Belov, Nanoscale, 2015.

19 S. Starikov, N. Y. Lopanitsyna, D. Smirnova and S. Makarov, Computational Materials Science, 2018, 142, 303-311.

20 S. Makarov, L. Kolotova, S. Starikov, U. Zywietz and B. Chichkov, Nanoscale, 2018.

21 H. Okamoto and T. Massalski, Bulletin of Alloy Phase Diagrams, 1983, 4, 190-198.

22 R. Méjard, A. Verdy, M. Petit, A. Bouhelier, B. Cluzel and O. Demichel, ACS Photonics, 2016, 3, 1482-1488. 
23 S. J. Plimpton, J. Comp. Phys., 1995, 117, 1.

24 E. D. Palik, Handbook of optical constants of solids, Academic press, 1998.

25 P. B. Johnson and R.-W. Christy, Physical review B, 1972, 6, 4370. 


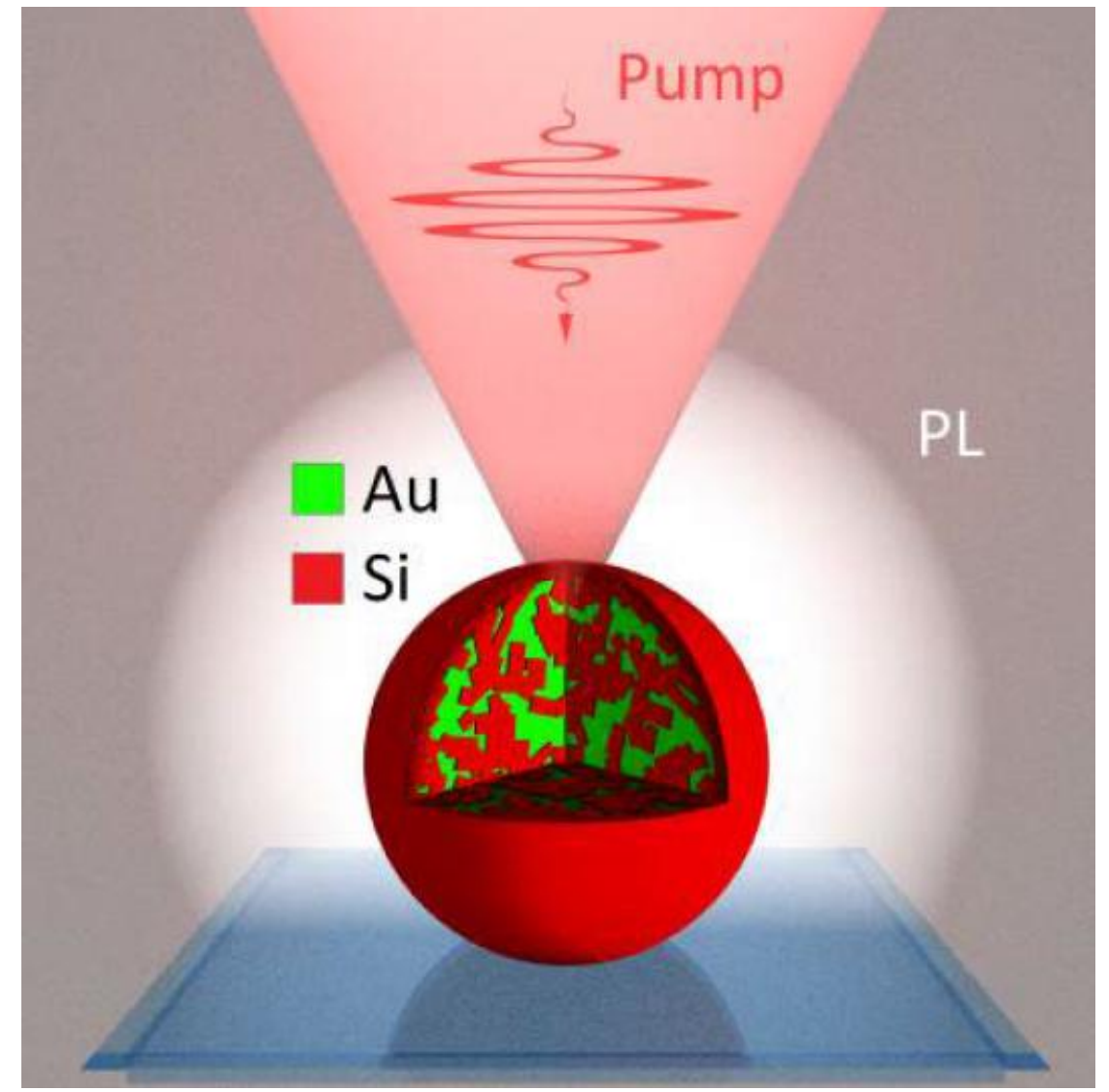

Fig. 1: Underlying concept: schematic of the hybrid Si/Au sponge nanoparticle on a silica substrate as a source of broadband photoluminescence due to the multiphoton absorption processes of femtosecond impulses. 


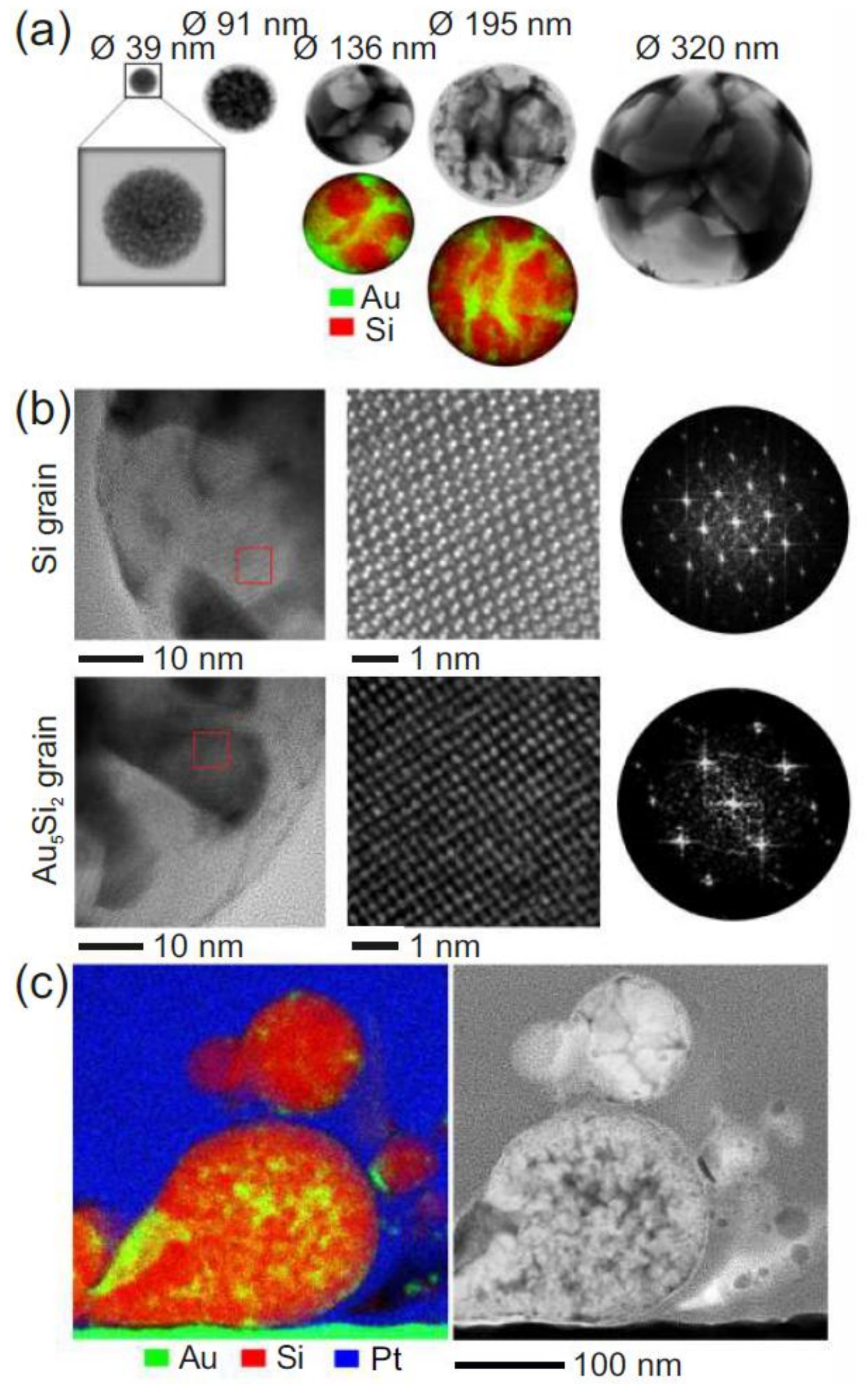

Fig. 2: (a) High Angle Annular Dark Field (HAADF) electron micrographs of Si/Au nanoparticles of different size with corresponding composition maps for the 136 and $195 \mathrm{~nm}$. (b) High-Resolution electron micrographs of Si-rich and Au-rich regions with a zoom in the red squares with the corresponding fast Fourier transformations. (c) Cross-sectional micrographs in spectral view and HAADF mode. 


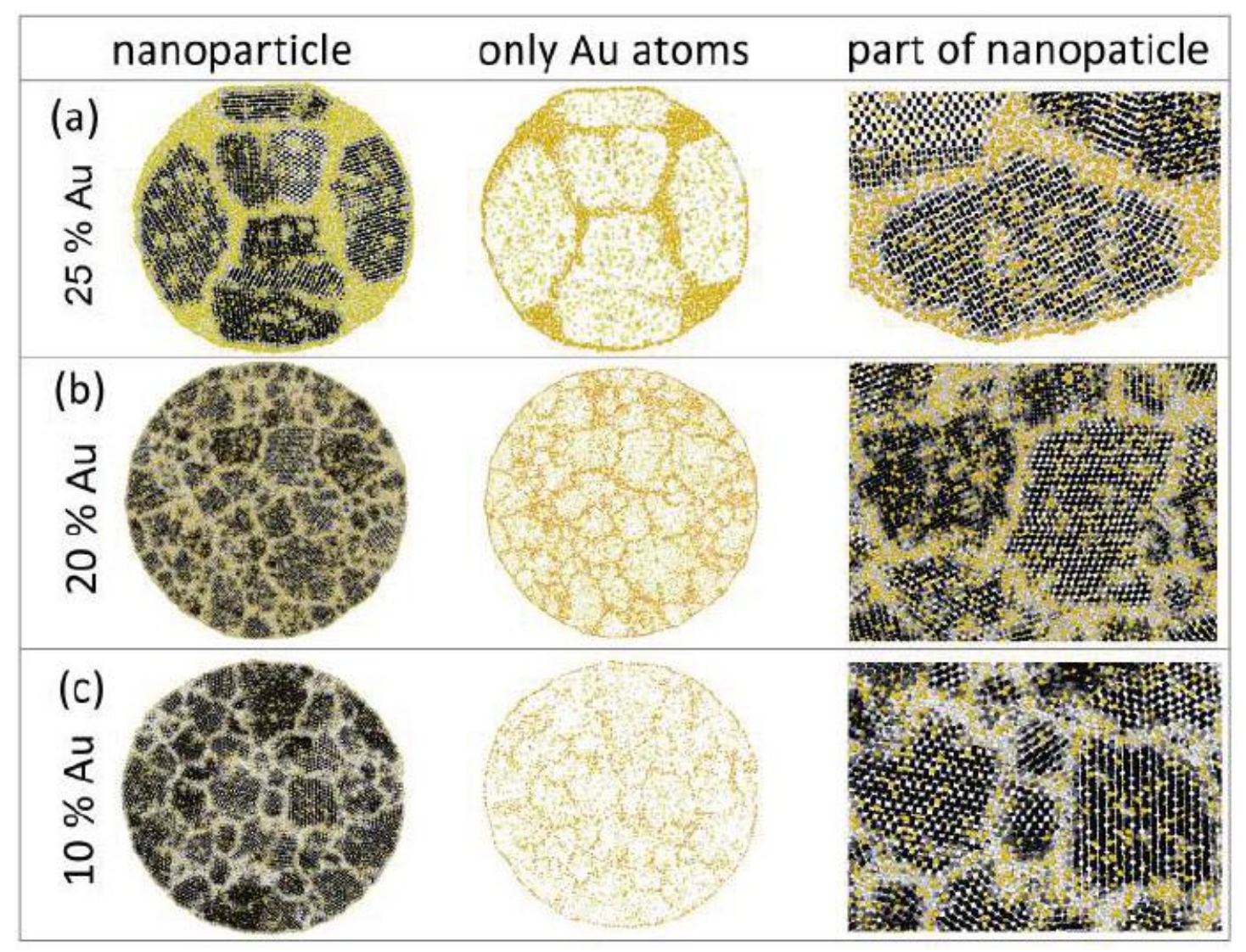

Fig. 3: Atomistic simulations of $\mathrm{Si} / \mathrm{Au} \mathrm{NPs}$ formation with various gold concentration, diameters and cooling rates - (a) $\mathrm{K}=0.01 \mathrm{~K} / \mathrm{ps}, \mathrm{D}=20 \mathrm{~nm}$; (b) $\mathrm{K}=0.1 \mathrm{~K} / \mathrm{ps}, \mathrm{D}=40 \mathrm{~nm}$; (c) $\mathrm{K}=0.35 \mathrm{~K} / \mathrm{ps}, \mathrm{D}=30 \mathrm{~nm}$. Silicon atoms are colored according to structure type, and the yellow atoms correspond to gold. 

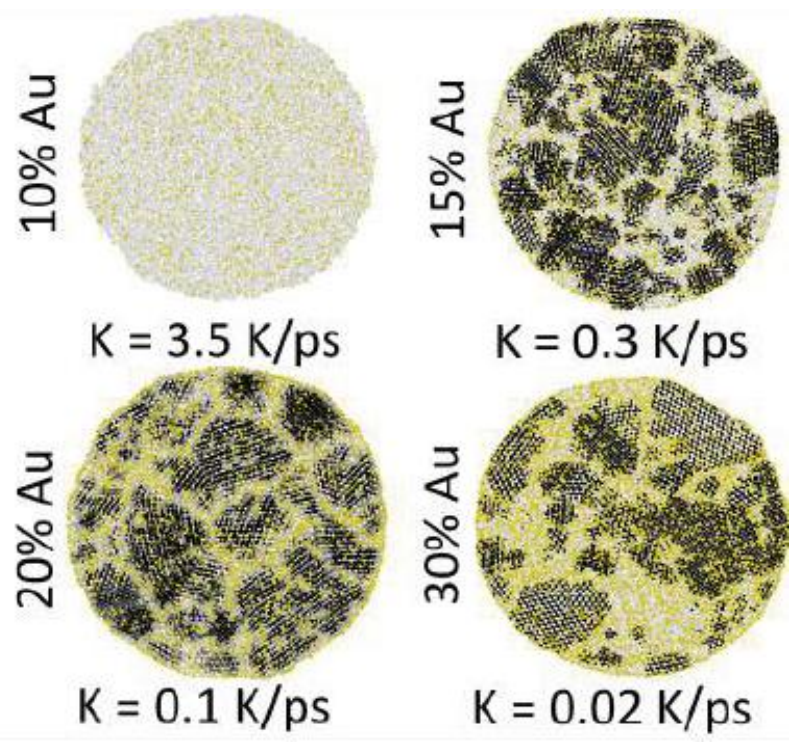

Fig. 4: $\mathrm{Si} / \mathrm{Au}$ NPs with the same size $\mathrm{D}=20 \mathrm{~nm}$ at different cooling rates for various $\mathrm{Au}$ concentration. Silicon atoms are colored according to structure type, and the yellow atoms correspond to gold. 


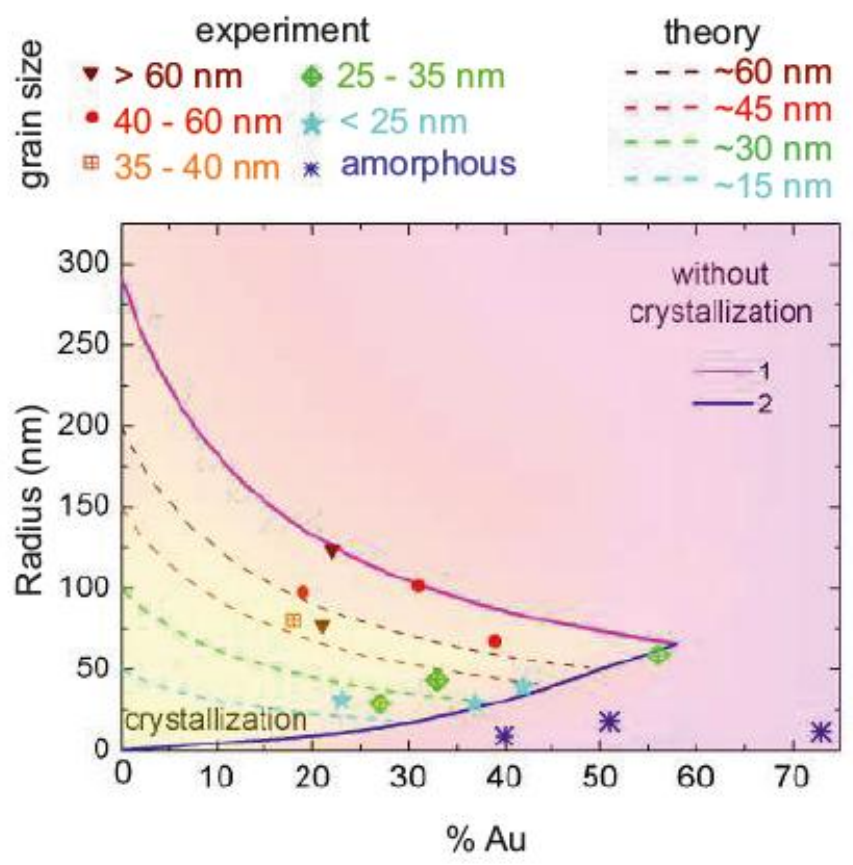

Fig. 5 The dependence of nanoparticle crystallization regions on its radius (R) and $\mathrm{Au}$ concentration calculated basing on the formulas (2) - line 1 and (3) - line 2 . The yellow region (small values of $\mathrm{R}$ and consentration) corresponds to nanoparticle crystallization by moving between substrates. The purple region (large values of $\mathrm{R}$ and consentration) corresponds to the remainder of the nanoparticle in the liquid state during the flight in air, implying further crystallization on a substrate. Dashed lines and points correspond the average grain size in nanoparticle at different cooling conditions estimated from the atomistic simulations (theory predictions) and experimental data, respectively. 
(a)
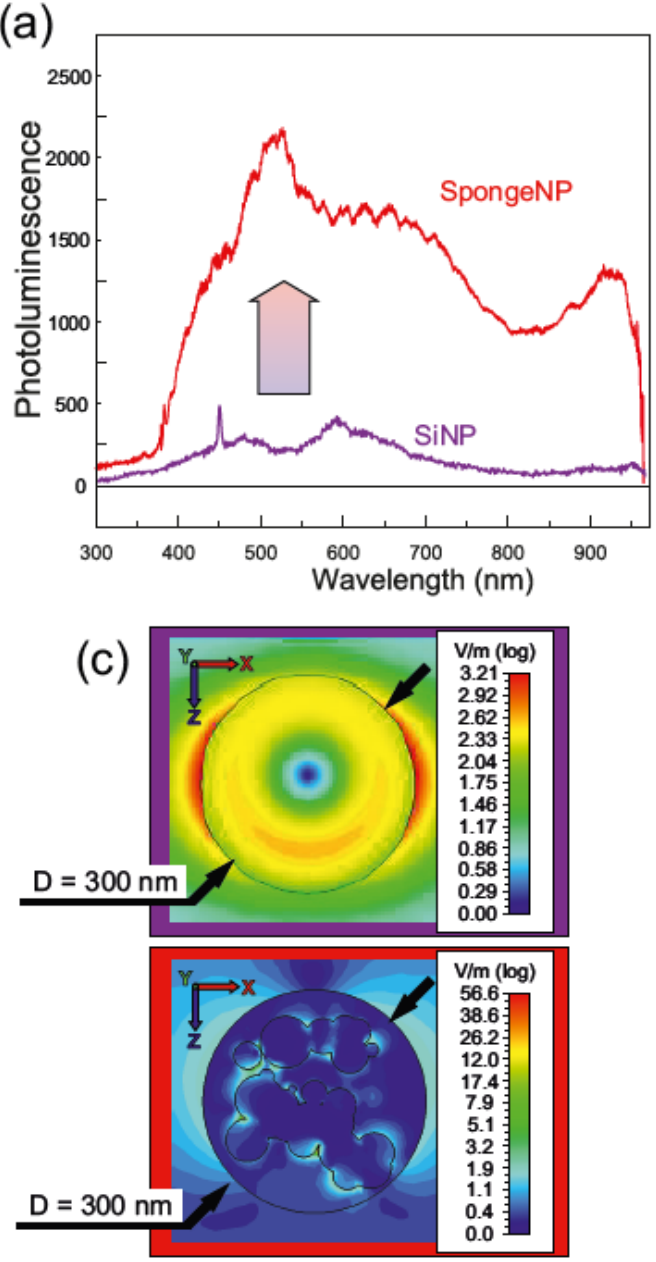

(b)

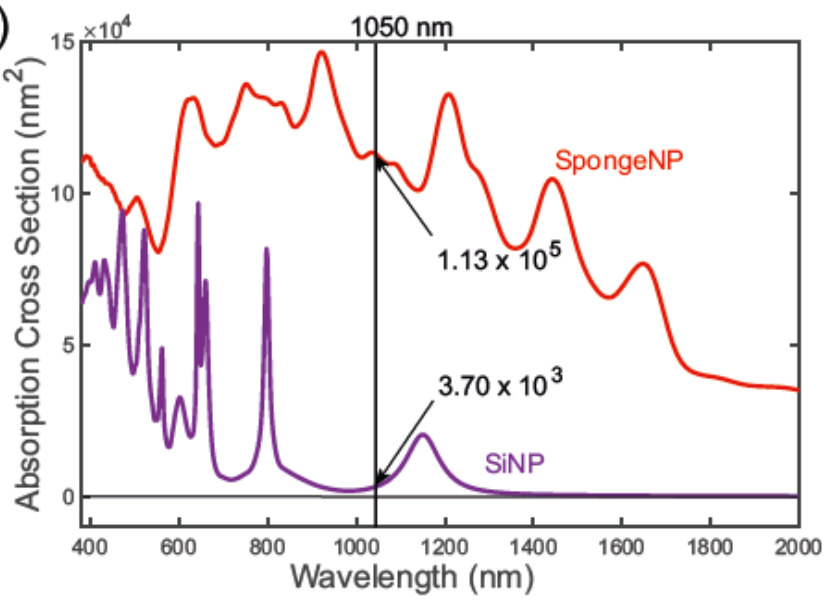

(d)

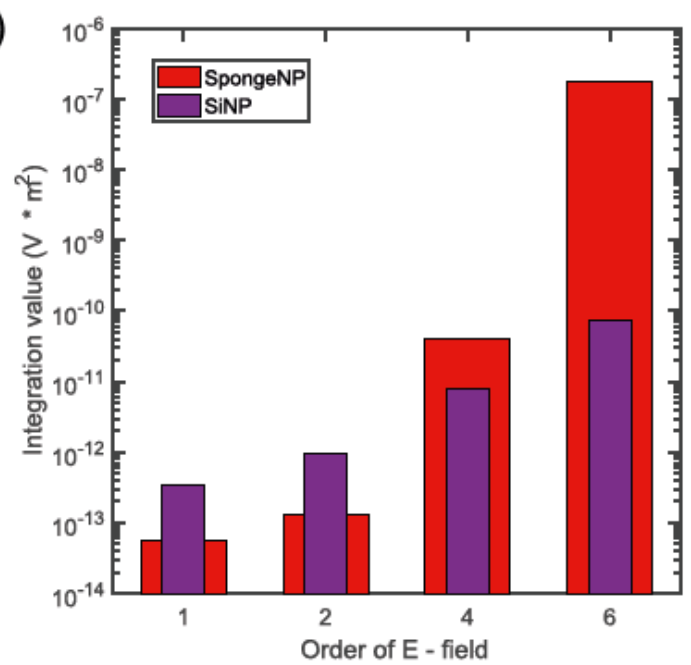

Fig. 6 Photoluminescent charachteristics of Si/Au NPs. (a) Photoluminescence of Si/Au (red line) and $\mathrm{Si}$ (blue line) nanoparticles. (b) Calculated absorption cross-sections for $\mathrm{Si} / \mathrm{Au}$ (red line) and $\mathrm{Si}$ (blue line) nanoparticles. (c) Calculated near-fields for Si/Au (red line) and $\mathrm{Si}$ (blue line) nanoparticles. (d) Diagram of integration value of average electric field for different degrees of electric field. 